\title{
BMC Medical Research Methodology reviewer acknowledgement 2015
}

Giulia Mangiameli

\section{Contributing reviewers}

The editors of BMC Medical Research Methodology would like to thank all our reviewers who have contributed to the journal in Volume 15 (2015).

\author{
Iosief Abraha
}

Italy

Michal Abrahamowicz

USA

Nima Aghaeepour

Canada

Mussie Akalu

USA

Hadi Alizadeh Noughabi

Iran

Mohamed Alosh

USA

Federico Ambrogi

Italy

Pavlina Andreeva-Gateva

Bulgaria

Judith Arnetz

USA

Jennifer Arney

USA

Kristopher Attwood

USA

Thomas Augustin

Germany

Erin Austin

USA

\author{
Steven Babbin \\ USA
}

Aïda Bafeta

France

Anna Bagnoli

UK

Samprit Banerjee

USA

Lola Baydala

Canada

Melanie Bell

USA

Antonia Bennett

USA

Vance Berger

USA

Regina Bernal

Brazil

Lucie Biard

France

Brad Biggerstaff

USA

Myriam Blanchin

France

Michael Blum

France
Amy Bodde
USA

Aline Boligon

Brazil

Lyndal Bond

Australia

Mohamed Boucekine

France

Michel Boudreaux

USA

James Boyd

Australia

Peter Bragge

Australia

Christian Brettschneider

Germany

Karl Broman

USA

Jenni Brooks

UK

Hilary Brown

Canada

Sylwia Bujkiewicz

UK

Peter Burgard

Germany 
Jonathan Burton

UK

Lisa Calderwood

UK

Marion Campbell

UK

Math Candel

Netherlands

Massimiliano Caporin

Italy

Brad Carlin

USA

Carme Carrion

Spain

An-Wen Chan

Canada

Yu-Mei Chang

Taiwan

Maggie Chen

Canada

Bingshu Chen

Canada

Xiwei Chen

USA

Yuhui Chen

USA

Fati Cheraghi

Iran

Francesco Chiappelli

USA

Jennifer Childs

USA

Kacper Chwialkowski

UK

Jérémie F. Cohen

France

Clare Coleman

Australia

Cindy Cooper

UK

Shannon Cope

Canada

Heather Cordell

UK
Enzo Coviello

Italy

Katelynn Crick

Canada

Joanna Crocker

UK

Rada Dagher

USA

Dong Dai

USA

Eleonora Dal Grande

Australia

Sayan Dasgupta

USA

Annemarie De Greeff

UK

Elizabeth Dean

USA

Salome Dell-Kuster

Switzerland

Jacques Demotes

France

Nandini Dendukuri

Canada

Sean Devlin

USA

Karla Diazordaz

UK

Joseph Dieleman

USA

Markus K. Diener

Germany

Kai Ding

USA

Shanshan Ding

USA

Jac Dinnes

UK

Sarah Donegan

UK

Carola Döpp

Netherlands

Vera Ehrenstein

Denmark
Deborah Ehrenthal USA

Marinus Eijkemans

Netherlands

Andreas Eiset

Denmark

Alan Ellis

USA

Alexander Engelhardt Germany

Jacques Estève

France

Paolo Eusebi

Italy

Caroline Fairhurst

UK

Hongbin Fang

USA

Yixin Fang

USA

Debra Fayter

UK

Lambert Felix

UK

Padhraig Fleming

UK

Dean Follmann

USA

Maria Joao Forjaz Spain

Ben Francis

UK

William Funk

USA

Daniel Gaile

USA

Paul Gallo

USA

Feng Gao

USA

Dana Garbarski

USA

Emily Geisen

USA 
Phil Gendall

New Zealand

Eveline Geubbels

Tanzania

Michael Gionfriddo

USA

Jean Golding

UK

Manuel Gomes

UK

Mithat Gonen

USA

Jessica Gorman

USA

Erika Graf

Germany

Teresa Greco

Italy

Mechthild M. Gross

Germany

Benjamin Guedj

France

Yi Guo

USA

Marianne Haapea

Finland

Rachael Hageman Blair

USA

Alix Hall

Australia

Nicholas Hamm

Netherlands

Sebastien Haneuse

USA

Timothy Hanson

USA

Jean-Benoit Hardouin

France

James Harrison

USA

Markus Hartmann

Germany

Ron Hays

USA
Sebastian Heidenreich

UK

Noor Heim

Netherlands

Günter Heimann

Switzerland

Daniel Heitjan

USA

Enrique Hernández-Lemus

Mexico

Ralf-Dieter Hilgers

Germany

John Hoey

Canada

Rebecca Holman

Netherlands

Hwanhee Hong

USA

Maria Horne

UK

Bo Hu

USA

Tania Huedo-Medina

USA

Alan Hutson

USA

Dan Jackson

UK

Martin Jenkins

UK

Prabhat Jha

Canada

Ming Ji

USA

Bin Jia

China

Xiaoqian Jiang

USA

Hua Jin

China

Rebecca Johnson

UK

David Johnson

USA
Hayley Jones

UK

Mona Kanaan

UK

Kush Kapur

USA

Monika Kastner

Canada

Athanassios Katsis

Greece

Ada Keding

UK

Luke Keele

USA

Paul Kelly

UK

Friederike Kendel

Germany

Antonia Keung

UK

Michael King

UK

Atle Klovning

Norway

Marija Kovandzic

UK

Tatsuki Koyama

USA

Walter Kremers

USA

Oliver Kuss

Germany

Samantha Lain

Australia

Christophe Lalanne

France

George Lambrou

Greece

Luís Lapão

Portugal

Nicholas Latimer

UK

Sebastien Laurent

France 
Quang Le

USA

Minji Lee

USA

Sunghee Lee

USA

Oliver Lee

USA

Boris Lemeshko

Russian Federation

Shanshan Li

USA

Megan Lim

Australia

Wan-Yu Lin

Taiwan

Joey Lin

USA

Aiyi Liu

USA

Shuling Liu

USA

Danping Liu

USA

Fang Liu

USA

Guohui Liu

USA

Per Liv

Sweden

Bronwyn Long

Australia

Mary Losch

USA

Thomas Love

USA

Tanzy Love

USA

Ray Lovett

Australia

Qingshu Lu

Singapore

Fabio Luciani

Australia
Karsten Lunze

USA

Sheng Luo

USA

Xiaoye Ma

USA

Malcolm Macleod

UK

Samuel Manda

South Africa

Chang Xuan Mao

China

Ronald Mataya

USA

Gregory Matthews

USA

Audrey Mauguen

France

Sally Mcclean

UK

Geoff Mclachlan

Australia

Sanjay Mehendale

India

Loren Mell

USA

Branko Miladinovic

USA

Christopher Miller

USA

Joanna Mills Flemming

Canada

Kristin Mmari

USA

Dylan Molenaar

Netherlands

Maureen Monaghan

USA

David Morgan

USA

Saman Muthukumarana

Canada

Laeth Nasir

USA
Hannelore Neuhauser

Germany

Cattram Nguyen

Australia

Pentti Nieminen

Finland

Sa-Aat Niwitpong

Thailand

Tanja Nordström

Finland

Eberechukwu Onukwugha

USA

Treena Orchard

Canada

Matthew Page

Australia

See-Tong Pang

Taiwan

Sameer Parpia

Canada

Sujata Patil

USA

Laure Perrier

Canada

Sydney Pettygrove

USA

Markus Pfirrmann

Germany

Ba' Pham

Canada

Siobhan Phillips

USA

Dawid Pieper

Germany

Melania Pintilie

Canada

Romain Pirracchio

USA

Maja Pohar Perme

Slovenia

Raphael Porcher

France

Bernardo Prado

USA 
Sandip Prasad

USA

Elizabeth Quon

USA

Daphna Ram

USA

Gowri Raman

USA

Rasika Rampatige

Australia

Marc Ratkovic

USA

Daniel Rigby

UK

Richard Riley

UK

Allysha Robinson

Thomas Rusch

Austria

Lila Rutten

USA

Mike Sachs

USA

Mohsen Sadatsafavi

Canada

Sandra Safo

USA

Rustam Salman

UK

Margaret Sampson

Canada

Enid Schatz

USA

Roberta Scherer

USA

Jonathan Schildcrout

USA

Björn Schreiweis

Germany

Shuan Seaman

UK

Véronique Sébille

France
Jadbinder Seehra

UK

Larissa Shamseer

Canada

Meiyu Shen

USA

Tsung-Jen Shen

Taiwan

James Sheppard

UK

Qiuling Shi

USA

Yu-Shan Shih

Taiwan

Abigail Shoben

USA

Haochang Shou

USA

Juned Siddique

USA

William Siero

Australia

Volkert Siersma

Denmark

Alla Sikorskii

USA

Mark Simmonds

UK

Becky Skidmore

Canada

Lindsey Smith

USA

Janet Smylie

Canada

Rebecca Stanley

Australia

Alisa Stephens Shields

USA

Adrienne Stevens

Canada

John Stevenson

USA

Ewout Steyerberg

Netherlands
Vicky Strauss

UK

Lorna Sweeney

UK

Yemisi Takwoingi

UK

Monica Taljaard

Canada

Yuanyuan Tang

USA

Johanna Taylor

UK

Steven Teerenstra

Netherlands

David Torgerson

UK

Annette Totten

USA

Shaun Treweek

UK

Javier Trujillano

Spain

Athanasios Tsalatsanis

USA

Konstantinos Tsilidis

Greece

Ruth Turley

UK

Elizabeth Turner

USA

Hannu Vähänikkilä

Finland

Jan Van De Kassteele

Netherlands

Johanna Van Der Lee

Netherlands

David Van Klaveren

Netherlands

Karen Van Leeuwen

Netherlands

Ravi Varadhan

USA

Monica Vasquez

USA 
Dinusha Vatsalan

Australia

Craig Velozo

USA

Areti Angeliki Veroniki

Canada

Wolfgang Viechtbauer

Netherlands

Byron Wallace

USA

Xiang Wan

Hong Kong

Handan Wand

Australia

Pengyuan Wang

USA

Zhen Wang

USA

Yaping Wang

USA

James Wason

UK

Lesley Weaver

USA

David Weiss

USA

Rolf Weitkunat

Switzerland

Ian R White

UK
Sarah White

UK

William Whiteley

UK

Andreas Wienke

Germany

Donald J Willison

Canada

Rosemary Wilson

Canada

Ed Wilson

UK

Julian Wolfson

USA

James Wright

Canada

Kath Wright

UK

Jianrong Wu

USA

Vicki Xafis

Australia

Fang Xiang

USA

Diqiong Xie

USA

Tu Xu

USA

Jose-Miguel Yamal USA
Wei Yang

USA

Hung-Wen Yeh

USA

Gui-Shuang Ying

USA

Jennifer Yost

Canada

Zhiying You

USA

Jihnhee Yu

USA

Ingeborg Zehbe

Canada

Steven Zeliadt

USA

Jing Zhang

USA

Zhongyang Zhang

USA

Daniel Zhao

USA

Yize Zhao

USA

Jiwei Zhao

USA

Yeying Zhu

Canada

Jeanette Ziegenfuss USA 